\title{
Early Stage Lymphedema in Breast Cancer Patient Detected by Indocyanine Green Lymphography but not by Lymphoscintigraphy: A Case Report
}

\author{
Jin A Yoon, M.D., Ph.D. ${ }^{1}$, Myung Jun Shin, M.D., Ph.D. ${ }^{1}$, Young Jin Choi, M.D., Ph.D. ${ }^{2}$, \\ Joo Hyoung Kim, M.D., Ph.D. ${ }^{3}$, Taewoo Kang, M.D., Ph.D. ${ }^{4}$, Heeseung Park, M.D., Ph.D. ${ }^{4}$ \\ ${ }^{1}$ Department of Rehabilitation Medicine, Pusan National University School of Medicine and Biomedical Research Institute, Pusan National University \\ Hospital, Busan; ${ }^{2}$ Department of Hemato-oncology, Pusan National University Hospital and Pusan National University School of Medicine, Busan; \\ ${ }^{3}$ Department of Plastic and Reconstructive Surgery and Biomedical Research Institute, Pusan National University Hospital, Busan; ${ }^{4}$ Department of \\ Surgery (Busan Cancer Center) and Biomedical Research Institute, Pusan National University Hospital, Busan, Korea
}

Secondary upper extremity lymphedema after lymph node dissection in breast cancer patients is a major complication affecting their quality of life. As lymphatic function is compromised before symptoms develop in most cases, early diagnosis and intervention before symptomatic manifestation is known to prevent severe progression of lymphedema. In this case report, we present a patient with secondary lymphedema, whose disease could be diagnosed at an early stage using indocyanine green (ICG) lymphography. The patient had mild edema of the left upper extremity that regressed with elevation, had normal lymph vessel distribution without dermal backflow pattern in lymphoscintigraphy. Her symptom improved after early prescription of complex decongestive physiotherapy. We conclude that ICG lymphography could be an effective diagnostic tool in addition to other imaging methods, for early diagnosis and intervention of lymphedema.

Key Words: Breast neoplasms, Breast cancer lymphedema, Indocyanine green

\section{INTRODUCTION}

The detection of secondary lymphedema has increased due to the advancement of breast cancer treatment. Therefore, early diagnosis and precise evaluation are necessary to minimize the progression of edema and prevent lymphatic drainage failure [1]. Currently, lymphoscintigraphy is mainly used for the detection of abnormal lymphatic flow, for which early diagnosis remains challenging [2]. Indocyanine green (ICG) lymphography, a new technique for imaging lymph vessels, can reveal lymphatic abnormalities at an early stage, possibly even before swelling becomes apparent [3]. Despite these advantages, it is currently only available at very few rehabilitation centers. In this case report, we present a patient with early diagnosis of secondary lymphedema by ICG lymphography.

\section{Correspondence: Heeseung Park, M.D., Ph.D.}

Department of Surgery, Busan Cancer Center, Pusan National University Hospital and Biomedical Research Institute, Pusan National University Hospital, 179 Gudeok-ro Seo-gu, Busan 49241, Korea

Tel: +82-51-240-7281, Fax: +82-51-240-7746, E-mail: paul0109@naver.com

This work was supported by clinical research grant from Pusan National University Hospital in 2020.

Received: Jul 11, 2019 Revised: Oct 2, 2019 Accepted: Oct 25, 2019

\section{CASE REPORT}

A 44-year-old woman visited our hospital and presented with swollen left upper extremity that persisted for 2 weeks, following a total left mastectomy and lymphatic dissection performed one year ago. On her first visit, the physical examination revealed enlargement of the circumference of the left upper extremity by $+1.6 \mathrm{~cm}$ above the elbow, $+1.2 \mathrm{~cm}$ below the elbow, and $+0.1 \mathrm{~cm}$ at the wrist and hand compared to the right side. During the initial visit, her neutrophil count, C-reactive protein titer and D-dimer were confirmed to be normal based on the findings of blood tests, and the possibility of infection was very small. By ultrasonography, a diffuse subcutaneous edema was observed around the elbow with no evidence of deep vein thrombosis.

Lymphoscintigraphy (GE xeleris, Milwaukee, USA) was performed with subcutaneous 99mTc injection into bilateral second and third interdigits ( $0.1 \mathrm{~mL}$ each, $0.4 \mathrm{~mL}$ in total, $80 \mathrm{MBq})$. On lymphoscintigraphy, no dermal back flow or cut-off flow were noted in the left upper extremity compared to those in the right side. Normal lymph flow and axillary lymph node activity revealed that there was no definite evidence of lymphedema in this study (Figure 1). 


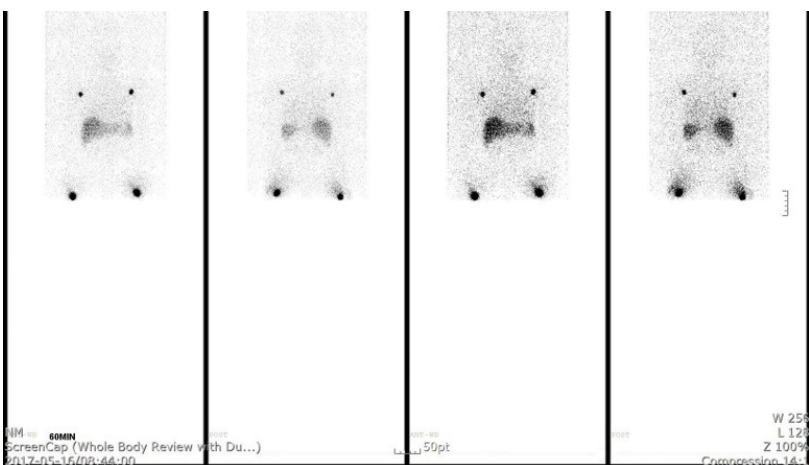

Figure 1. Lymphoscintigraphy showing normal lymphatic flow and axillary lymph node in both upper extremities.

For further evaluation, ICG was injected subcutaneously $(0.2 \mathrm{~mL}$ into left interdigits) to image the superficial lymphatics and dermal backflow pattern by ICG lymphography. Images of the left arm were acquired using a charge-coupled device (CCD) camera 5-, 30-, 60-, 120-, and 180 minutes and 24 hours after injection. The lymphography showed several splash patterns and tortuous lymphatic channels, indicating early stage mildly impaired lymph flow at the left dorsum of the hand within a few minutes after injection (Figure 2). In addition, a stardust pattern due to extravasation of lymph fluid was observed in the left forearm and a mixed splash and diffuse pattern was noted at a more proximal part, thereby indicating severely damaged lymph flow (Figure 3).

After confirming abnormal lymphatic flow and dermal backflow patterns using ICG lymphography, it was established to be clinically evident lymphedema, indicating a critical time point to initiate treatment. We were able to describe the need for active complex decongestive physiotherapy to the patients, and treatment such as education of manual lymphatic draining, wearing compressive stocking, and pneumatic compression was applied as early intervention. One month after the commencement of treatment, the difference in circumference of the upper extremity decreased to $0.5 \mathrm{~cm}$ above and below elbow, and the patient was able to continue treatment with good compliance. This case study was approved by our Institutional Review Board, and the requirement for written consent was waived (IRB No. 1909-023-083).

\section{DISCUSSION}

In this case study, early stage lymphedema was diagnosed by ICG

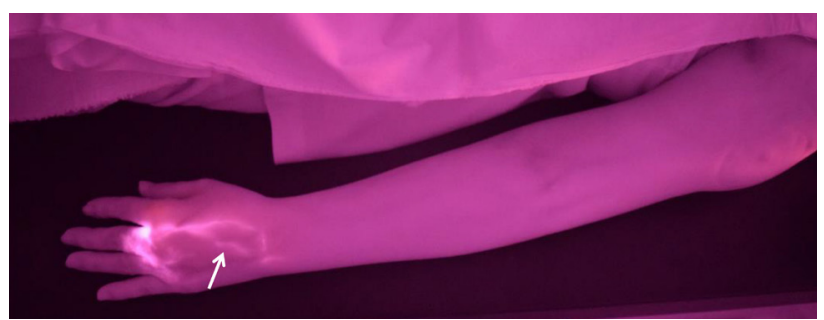

Figure 2. ICG lymphography 5 minutes after injection, indicating splash pattern at left dorsum of hand.

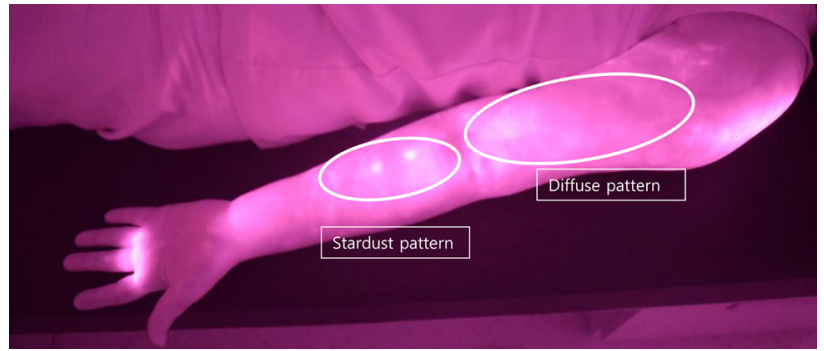

Figure 3. ICG lymphography 24 hours after injection, indicating stardust pattern in left forearm and diffuse pattern in left upper arm.

lymphography, which allowed the patient to select the management as and when necessary. As lymphedema can induce not only pathologic problems like recurrent cellulitis but also cosmetic concerns influencing severe functional disabilities in breast cancer patients, early diagnosis of secondary lymphedema is vital for its treatment prognosis [4]. However, detecting lymphedema at an early stage is challenging, as its manifestations vary greatly among patients and the edema usually initiates gradually, long after lymphatic vessel disruption.

Patient history, physical examination, imaging of soft tissue, lymph vessel, and lymph node, and measuring of volume are among the commonly used tools to evaluate lymphedema. For clinical lymphedema staging, Campisi reported the importance of early diagnosis at stage $1 \mathrm{~A}$ or $1 \mathrm{~B}$, no edema or edema that regressed with elevation despite the presence of lymphatic dysfunction [5]. To know the critical point of initiation of treatment, it is not suitable to apply repetitive lymphoscintigraphy for routine follow-up due to its radiation exposure.

ICG lymphography is a non-invasive, non-radioactive tool to evaluate lymphatic function. Although it cannot be used to observe lymph vessels deeper than $2-3 \mathrm{~cm}$. ICG lymphography is a useful tool to evaluate the lymphatic circulatory condition by dermal backflow stage and lymph pump function by lymph transportation quantifica- 
tion [6]. Specific dermal backflow patterns are observed as linear patterns progress to splash, stardust and diffuse patterns appearing in this order with severity of edema. In particular, it can detect abnormal conditions of the lymphatic circulation before edema becomes clinically evident. As seen in our patient, these splash patterns are indicative of a reversible lymphatic disorder and would indicate a critical time point at which to start appropriate management [7].

Mihara et al. [8] reported ICG lymphography to be superior to lymphoscintigraphy for the diagnostic imaging of early lymphedema, as the diagnostic sensitivity was 1 by ICG lymphography and 0.62 by lymphoscintigraphy. There are also marked differences in the direct observation of the lymphatic system between these two methods. In ICG lymphography, retained fluid is detectable without background noise because there is no endogenous fluorescence in the near infrared band used for the detection of ICG, thereby increasing the accuracy [8]. In addition, when there is no definite lymphatic flow obstruction or dermal backflow, it is possible to overlook decreased lymphatic flow with qualitative lymphoscintigraphy [9].

Likewise, our case showed normal lymph flow and axillary lymph node activity in lymphoscintigraphy but various abnormal dermal backflows in ICG lymphography, which was proven to be an indispensable diagnostic tool for our patient. As all diagnostic methods have both strengths and weaknesses, combining diagnostic tools for evaluation of the lymphatic system might be advantageous. Regarding the pattern of dermal backflow distribution, our patient showed more severe pattern at proximal part of the limb (stardust and diffuse patterns) compared to the distal part (splash pattern). This dermal backflow pattern was previously described, wherein the linear pattern was found less frequently and dermal backflow pattern progressed to the stardust or diffuse patterns in the proximal part, like our case [7]. In addition, confirming various dermal backflow patterns in relation to the body part is another advantage of ICG lymphography which can provide additional information of which body part to focus on during lymphedema management, such as manual lymphatic drainage.

Although standardized performance metrics are necessary, ICG lymphography has various advantages. As the incidence of mild adverse reactions was reported to be $0.05 \%$ during intravenous administration [10], it would be lower, and therefore safer for subcutaneous injection. However, it is currently not available as a diagnostic tool due to the absence of appropriate medical charge and the equipment not yet being introduced in Korean healthcare settings. In this case report, we were able to make an early diagnosis of lymphedema and guide decisions to adjust lymphedema treatment by using ICG lymphography. With greater implementation efforts, ICG lymphography could become an essential clinical diagnostic tool for early diagnosis of lymphedema and staging. Considering the usefulness of ICG lymphography in early diagnosis of lymphedema, appropriate clinical settings are required for this to be implemented in Korea.

\section{CONFLICT OF INTEREST}

The authors declare that they have no competing interests.

\section{REFERENCES}

1. Warren AG, Brorson H, Borud LJ, Slavin SA. Lymphedema: a comprehensive review. Ann Plast Surg 2007;59:464-72.

2. Zhibin Y, Quanyong L, Libo C, Jun Z, Hankui L, Jifang Z, et al. The role of radionuclide bone scintigraphy in fibrous dysplasia of bone. Clin Nucl Med 2004;29:177-80.

3. Yamamoto T, Matsuda N, Doi K, Oshima A, Yoshimatsu H, Todokoro $\mathrm{T}$, et al. The earliest finding of indocyanine green lymphography in asymptomatic limbs of lower extremity lymphedema patients secondary to cancer treatment: the modified dermal backflow stage and concept of subclinical lymphedema. Plast Reconstr Surg 2011;128:314-21e.

4. Finnane A, Hayes SC, Obermair A, Janda M. Quality of life of women with lower-limb lymphedema following gynecological cancer. Expert Rev Pharmacoecon Outcomes Res 2011;11:287-97.

5. Campisi C, Boccardo F. Microsurgical techniques for lymphedema treatment: derivative lymphatic-venous microsurgery. World J Surg 2004;28:609-13.

6. Unno N, Inuzuka K, Suzuki M, Yamamoto N, Sagara D, Nishiyama $\mathrm{M}$, et al. Preliminary experience with a novel fluorescence lymphography using indocyanine green in patients with secondary lymphedema. J Vasc Surg 2007;45:1016-21.

7. Yamamoto T, Narushima M, Doi K, Oshima A, Ogata F, Mihara M, et al. Characteristic indocyanine green lymphography findings in lower extremity lymphedema: the generation of a novel lymphedema severity staging system using dermal backflow patterns. Plast 
Reconstr Surg 2011;127:1979-86.

8. Mihara M, Hara H, Araki J, Kikuchi K, Narushima M, Yamamoto T, et al. Indocyanine green (ICG) lymphography is superior to lymphoscintigraphy for diagnostic imaging of early lymphedema of the upper limbs. PloS One 2012;7:e38182.

9. Dalia RM, Martins GRP, Barbosa R, Lima CF, Siqueira CF. Qualita- tive and quantitative lymphoscintigraphy in the evaluation of lower limbs lymphedema. Braz Arch Biol Technol 2005;48:159-62.

10. Marshall MV, Rasmussen JC, Tan IC, Aldrich MB, Adams KE, Wang X, et al. Near-infrared fluorescence imaging in humans with indocyanine green: a review and update. Open Surg Oncol J 2010;2: $12-25$. 